\title{
The critical points in induction of experimental autoimmune uveitis
}

\author{
Aneta Klimova ${ }^{a}$, Petra Seidler Stangovaa ${ }^{a}$ Petra Svozilkovaa , John V. Forrester ${ }^{\mathrm{b}, \mathrm{c}}$, Izabela Klaska ${ }^{\mathrm{b}}$, Jarmila Heissigerova ${ }^{\mathrm{a}}$
}

Background. Autoimmune uveitis is a leading cause of visual impairment in developed countries in patients of working age. Animal models of experimental autoimmune uveitis (EAU) have been established to serve as a useful template for novel therapeutic approaches.

Methods. Experimental autoimmune uveitis is induced in C57BL/6 mice by subcutaneous application of interphotoreceptor retinoid binding protein in complete Freund's adjuvant and pertussis toxin. Clinical and histological grading is used to assess the inflammation intensity of EAU.

Results. The protocol of induction of EAU in mice hides several important aspects, which are crucial for developing the disease. These details have to be addressed to ensure reproducible disease induction. We describe our experience in establishing the model by pointing out the critical steps in EAU protocol which we found important.

Conclusion. The mouse model of EAU has practical value for preclinical studies, is robust and well established. However, the induction of inflammation of the eye can be quite challenging when important details of the protocol are not recognized and adhered to.

Key words: experimental autoimmune uveitis, C57BL/6 mouse strain, mouse model, induction protocol, chronic posterior autoimmune uveitis

Received: May 16, 2015; Accepted with revision: November 2, 2015; Available online: November 10, 2015

http://dx.doi.org/10.5507/bp.2015.056

${ }^{a}$ Department of Ophthalmology, First Faculty of Medicine, Charles University in Prague and General University Hospital in Prague, Czech Republic

'Section of Immunology and Infection, Institute of Medical Sciences, University of Aberdeen, United Kingdom 'Immunology and Virology Program, Centre for Ophthalmology and Visual Science, The University of Western Australia, Crawley, Western Australia, Australia and Centre for Experimental Immunology, Lions Eye Institute, Nedlands, Western Australia, Australia Corresponding author: Aneta Klimova, e-mail:aneta.klimova@volny.cz

\section{INTRODUCTION}

Uveitis is an intraocular inflammation affecting mostly people of working age. In spite of expanding therapeutic possibilities $10 \%$ of patients have severe visual handicap ${ }^{1}$. Clinical studies are difficult to perform for ethical reasons, therefore animal models were developed. Models of experimental autoimmune uveoretinitis are representative for various forms of human intraocular inflammation and serve as a useful template for novel immunotherapeutical approaches.

Mouse models of experimental autoimmune uveitis (EAU) have been established by Rachel Caspi more than 20 years ago ${ }^{2,3}$. After rat, rabbit, guinea pig and primate uveitis models the mouse model is most sophisticated and versatile. Thanks to the many genetically manipulated strains and variety of reagents, it is possible to perform research in the mouse model to extent, which is impossible to achieve in other species ${ }^{4}$. The development of experimental autoimmune uveitis helps to understand the mechanisms of autoimmune disease and to find more specific treatment with fewer side effect ${ }^{5}$.

Although the model of EAU is quite robust, the protocol of induction has to be followed precisely. Successful induction of EAU is based on careful laboratory preparation of the emulsified antigen as well on proper subcutaneous application of the antigen emulsion.

\section{MATERIALS AND METHODS}

The uveitis in mice is induced by subcutaneous application of the retinal autoantigen interphotoreceptor retinoid binding protein (IRBP) or its uveitogenic peptide, available in several versions according to a mouse strain ${ }^{6}$ in complete Freund's adjuvant (CFA). The reactivity of immune system is furthermore enhanced by intraperitoneal application of pertussis toxin (PT) depending on the mouse strain.

The susceptibility to IRBP induced EAU model varies with mouse strain, the most susceptible is B10.RIII, followed by B10.A. The C57BL/6 strain is only moderately susceptible ${ }^{4}$, but provides mild posterior uveitis, which has a longer duration of the disease and the unaffected anterior eye segment allows to examine retina clearly. C57BL/6 is a common mouse strain available in knockout, transgenic and gnotobiotic form. Some strains (e.g. BALB/c) are resistant to EAU ( ref. $^{7}$ ).

We used inbred female mice strain C57BL/6 6 to 8 weeks old, since we wished to induce a chronic mild inflammation, mainly involving the posterior segment of the eye ${ }^{8}$. This imitates most closely posterior uveitis in humans and is therefore most appropriate to test new therapeutical strategies. Noticeably, for induction of experimental autoimmune uveitis is appropriate to use the mouse substrain C57BL/6J. The substrain C57BL/6N 
carries the rd8 retinal degeneration mutation and mice congenitally lack photoreceptor cells ${ }^{7}$.

The peptide inducing experimental autoimmune uveitis in C57BL/6 mouse strain, also called retinol-binding protein 3 precursor fragment ([Homo sapiens] $\mathrm{H} 2 \mathrm{~N}$ GPTHLFQPSLVLDMAKVLLD-OH, New England Peptide, Gardner, MA, USA) is commercially prepared as a fine powder. We recommend preparing the peptide emulsion in the original plastic container with the appropriate dose of the protein in milligrams since freeze dried peptide adheres readily to surfaces due to electrostatic effects and so is difficult to weigh to the correct amount. Dimethyl sulphoxide (DMSO, Sigma Aldrich, St. Louis, MO, USA) is used to dissolve the IRBP. This solution is further diluted by sterile distilled water, slowly in 3 steps. After each step the solution centrifuged for at least $3 \mathrm{~min}$ at $500 \mathrm{rpm}$. This produces a clear solution without particles or precipitates. The IRBP solution can be stored in freezer under $-20{ }^{\circ} \mathrm{C}$ for several months or longer.

The IRBP solution is mixed 1:1 with complete Freund's adjuvant (CFA, Sigma Aldrich, St. Louis, MO, USA), which can be strengthened by additional Mycobacteria tuberculosis either by adding 25 milligrams of heat-dried H37RA Mycobacteria tuberculosis organisms in $10 \mathrm{~mL}$ of CFA, containing $1 \mathrm{mg}$ of Mycobacterium tuberculosis in $1 \mathrm{~mL}$. The other possibility, which we prefer, is to remove the top $7 \mathrm{~mL}$ of pure oil from the $10 \mathrm{~mL}$ vial of CFA after sedimentation of Mycobacteria at the bottom of the vial. With first method we reach the concentration of Mycobacterium tuberculosis in oil $3.5 \mathrm{mg} / \mathrm{mL}$, with the second $3.3 \mathrm{mg} / \mathrm{mL}$.

We have found that a consistently reproducible emulsion depends on effective mixing of the suspension. The final product should be uniform (no separation of phases), white, stiff and viscous ${ }^{9}$ to stimulate a strong antigen response in mice. We use a plastic tube (Tub PTFE 2xHub, Hamilton Company, Reno, NV, USA) connected on both sides to glass, $1 \mathrm{~mL}$ volume, Hamilton syringes. It is recommended to use glass syringes, since equivalent 1 mL plastic (insulin) syringes are not PTFE (polytetrafluor ethylene)-manufactured and particles precipitate on the plastic surface, thus losing material. The syringe-hub-syringe must be air free to avoid a froth forming. Thorough manual mixing takes around 15 minutes (equivalent to 1000 passes through the syringes). Ultrasonic emulsification mixing ${ }^{10}$ reduces the time at this stage but requires short pulse of ultrasound to avoid damaging the peptide. The quality of emulsification is tested by placing a drop of emulsion on a cold water surface (a well-prepared emulsion will not spread and remains as droplets).

Subcutaneous application of $50 \mu \mathrm{L}$ of emulsion ( $500 \mu \mathrm{g}$ of IRBP per mouse) in each hind leg is performed using a short intradermal needle (BD Microlance Hypodermic Needle $26 \mathrm{G}$ x 3/8“). A shorter needle provides more comfortable subcutaneous instillation. A small uniform swelling will appear under the skin. The needle should be held in position under the skin for 2-3 s before removal so as to avoid backflush of emulsion. The subcutaneous swelling should remain palpable at least 2 weeks.
The injury of adjacent muscle may be severe and must be avoided by ensuring that the injection is correctly placed subcutaneously and not intramuscularly.

Since the in vivo procedure has to be performed carefully and precisely and may be moderately painful we prefer to work with mice under short mild anesthesia and analgesia by ketamine intraperitoneally in dose $2 \mathrm{mg} / \mathrm{kg}$ (Narkamon $50 \mathrm{mg} / \mathrm{mL}$, Bioveta, Nitra, Slovakia).

Disease induction is optimized by intraperitoneal application of 0.5-2 $\mu \mathrm{g}$ PT per mouse (List Biologicals, Campbell, CA, USA) at the day of induction. Lyophilized PT from Bordetella pertussis (stored in $4{ }^{\circ} \mathrm{C}$ ) is dissolved in sterile distilled water, and can be stored in $4{ }^{\circ} \mathrm{C}$. Immediately before administration PT is further diluted in cold PBS (phosphate buffered saline), transported on ice, but warmed to room temperature for the intraperitoneal injection. In general, this attention to detail in preparing the PT is very important since its potency for experimental autoimmune uveitis may be diminished. Similar observations have been made in the induction of experimental autoimmune encephalomyelitis (EAE) (ref. ${ }^{11}$ ).

The experimental use of animals was approved by The Commission for Animal Welfare of the First Faculty of Medicine of Charles University in Prague, Czech Republic, and the Ministry of Education, Youth and Sports according to animal protection laws. All the procedures were approved by the animal experimentation review committee.

\section{RESULTS AND DISCUSSION}

By following the above protocol the EAU in C57BL/6 mice is induced, which is normally a mild chronic disease of low intensity of inflammation. The intensity of inflammation varies from lab to lab. On average grades severity varies between grades 1-2 [2.13 (ref. ${ }^{12}$ ), between 1 and 2 (ref. $\left.{ }^{8}\right), 1.2$ (ref. $\left.{ }^{13}\right), 1.63$ (ref. $\left.{ }^{14}\right)$.] In our experience, using the above protocol the mean histological score is 2.0.

Even when following the above procedure precisely the incidence of disease can be variable. Other factors which affect this are age and sex. We use 6 to 8 weeks old mice as in other reports, while Xu et al. $\left(\right.$ ref. $^{8}$ ) used 8 to 12 week old mice. In general, female mice are preferred since autoimmune diseases are more prevalent in females. There are no publications comparing the intensity of uveitis between male and female mice.

The susceptibility of a specific mouse strain to induction of EAU may also vary with the housing conditions of mice. Chronically stressed mice (elevated corticosteroid levels suppress the inflammation) or animals with acute disease (elevated circulating interferons) may fail to develop EAU ( ref. $\left.^{7}\right)$.

A complication of oily adjuvant instillation is injection site ulceration ${ }^{15}$. These usually develop 2 to 3 weeks after injection and have been reported in induction of EAE and other models. Frequency of occurrence varies from $0.5 \%$ to $20 \%$ of animals. Most ulcers are small (up to $4 \mathrm{~mm}$ in size) but rarely large confluent ulcers may 
occur. Ulcer development is reduced by systemic immunosuppressive therapy and there is no correlation between ulcers formation and intensity of inflammation (personal observation).

\section{CONCLUSION}

A reliable, standardized protocol and procedure for induction would be valuable for its further use in research on disease pathogenesis and treatment. The interpretation of the results should take into account the variation in protocol and procedure used in each experiment. It is therefore important to include the possible variation in the evaluation of the results.

\section{ABBREVIATIONS}

EAU, Experimental autoimmune uveitis; IRBP, Interphotoreceptor retinoid binding protein; CFA, Complete Freund's adjuvant; PT, Pertussis toxin; EAE, experimental autoimmune encephalomyelitis.

Acknowledgement: This paper was supported by research project from the Ministry of Heath of the Czech Republic IGA MZ CR NT/14017-3/2013 and SVV UK 260148/2015.

Author contribution: AK: manuscript writing and literature search, PSS: data collection, PS: study design and manuscript writing, JVF, IK: model development support, JH: study design and manuscript writing.

Conflict of interest statement: The authors declare there are no conflicts of interest regarding the publication of this article.

\section{REFERENCES}

1. Durrani OM, Meads CA, Murray PI. Uveitis: a potencially blinding disease. Ophthalmologica 2004;218:223-36.

2. Caspi RR, Roberge FG, Chan CC, Wiggert B, Chader GJ, Rozenszajn LA, Lando Z, Nussenblatt RB. A new model of autoimmune disease. Experimental autoimmune uveoretinitis induced in mice with two different retinal antigens. J Immunol 1988;140:1490-5.

3. Caspi RR, Chan CC, Wiggert B, Chader GJ. The mouse as a model of experimental autoimmune uveoretinitis (EAU). Curr Eye Res 1990; 9(Suppl.):169-74.

4. Caspi RR. Animal models of autoimmune and immune-mediated uveitis. Drug Discov Today Dis Mod 2006;3:3-10.

5. Klimova A, Seidler Stangova P, Heissigerova J, Svozilkova P, Kucera T. Mycophenolate mofetil and cyclophosphamide treatments suppress inflammation intensity in an experimental model of autoimmune uveitis. Folia Biol (Praha) 2014;60:228-34.

6. Caspi RR, Silver PB, Luger D, Tang J, Cortes LM, Pennesi G, Mattapallil MJ, Chan CC. Mouse models of experimental autoimmune uveitis. Ophthalmic Res 2008;40:169-74.

7. Agarwal RK, Silver PB, Caspi RR. Rodent models of experimental autoimmune uveitis. Methods Mol Biol 2012;900:443-69.

8. Xu H, Koch P, Chen M, Lau A, Reid DM, Forrester JV. A clinical grading system for retinal inflammation in the chronic model of experimental autoimmune uveoretinitis using digital fundus images. Exp Eye Res 2008;87:319-26.

9. Stromnes IM, Goverman JM. Active induction of experimental allergic encephalomyelitis. Nat Protoc 2006;4:1810-9.

10. Maatta JA, Nygardas PT, Hinkkanen AE. Enhancement of experimental autoimmune encephalomyelitis severity by ultrasound emulsification of antigen/adjuvant in distinct strains of mice. Scand J Immunol 2000;51:87-90.

11. Bernard CC, Carnegie PR. Experimental autoimmune encephalomyelitis in mice: immunologic response to mouse spinal cord and myelin basic proteins. J Immunol 1975;114: 1537-40.

12. Oh JY, Kim TW, Jeong HJ, Lee HJ, Ryu JS, Wee WR, Heo JW, Kim MK. Intraperitoneal infusion of mesenchymal stem/stromal cells prevents experimental autoimmune uveitis in mice. 2014;2014:624640. doi: $10.1155 / 2014 / 624640$.

13. Keino $H$, Kezuka T, Takeuchi M, Yamakawa N, Hattori T, Usui M. Prevention of experimental autoimmune uveoretinitis by vasoactive intestinal peptide. Arch Ophthalmol 2004;122:1179-84.

14. Kitamei H, Kitaichi N, Yoshida K, Nakai A, Fujimoto M, Kitamura M, Iwabuchi K, Miyazaki A, Namba K, Ohno S, Onoé K. Association of heat shock protein 70 induction and the amelioration of experimental autoimmune uveoretinitis in mice. Immunobiology 2006;212:11-8.

15. Whitehouse M. Oily adjuvants and autoimmunity: now time for reconsideration? Lupus 2012;21:217-22. 